\title{
Complex II inhibition by 3-NP causes mitochondrial fragmentation and neuronal cell death via an NMDA- and ROS-dependent pathway
}

\author{
G Liot $^{1}$, B Bossy ${ }^{1,2}$, S Lubitz ${ }^{2}$, Y Kushnareva ${ }^{1}$, N Sejbuk ${ }^{1}$ and E Bossy-Wetzel ${ }^{\star, 1,2}$
}

\begin{abstract}
Mitochondrial respiratory complex II inhibition plays a central role in Huntington's disease (HD). Remarkably, 3-NP, a complex II inhibitor, recapitulates HD-like symptoms. Furthermore, decreases in mitochondrial fusion or increases in mitochondrial fission have been implicated in neurodegenerative diseases. However, the relationship between mitochondrial energy defects and mitochondrial dynamics has never been explored in detail. In addition, the mechanism of neuronal cell death by complex II inhibition remains unclear. Here, we tested the temporal and spatial relationship between energy decline, impairment of mitochondrial dynamics, and neuronal cell death in response to 3-NP using quantitative fluorescence time-lapse microscopy and cortical neurons. 3-NP caused an immediate drop in ATP. This event corresponded with a mild rise in reactive oxygen species (ROS), but mitochondrial morphology remained unaltered. Unexpectedly, several hours after this initial phase, a second dramatic rise in ROS occurred, associated with profound mitochondrial fission characterized by the conversion of filamentous to punctate mitochondria and neuronal cell death. Glutamate receptor antagonist AP5 abolishes the second peak in ROS, mitochondrial fission, and cell death. Thus, secondary excitotoxicity, mediated by glutamate receptor activation of the NMDA subtype, and consequent oxidative and nitrosative stress cause mitochondrial fission, rather than energy deficits per se. These results improve our understanding of the cellular mechanisms underlying HD pathogenesis.
\end{abstract}

Cell Death and Differentiation (2009) 16, 899-909; doi:10.1038/cdd.2009.22; published online 20 March 2009

Huntington's disease (HD) is a fatal progressive neurodegenerative disorder with autosomal dominant inheritance. An abnormal CAG expansion coding for a polyglutamine stretch in the $\mathrm{N}$-terminal region of the huntingtin gene causes HD. Disease results when the polyglutamine stretch contains 40 or more residues, and repeats of 36-39 residues have reduced penetrance. The clinical symptoms of HD include progressive motor, cognitive, and emotional deficits due to the changes in the cortex and striatum. How mutant huntingtin ( $\mathrm{mtH} t \mathrm{t})$ triggers neurodegeneration is not clear. Among the proposed mechanisms are transcriptional dysregulation, axonal and dendritic transport defects, protein aggregation, and excitotoxic pathways mediated by glutamate receptors.

In addition to these pathways, there is strong evidence that deficits in energy metabolism and mitochondria play a pivotal role in HD pathogenesis. ${ }^{1-6}$ For example, brain tissue of HD patients $^{1,2}$ and transgenic mice expressing the $\mathrm{mtHtt}$ gene ${ }^{5}$ exhibit reduced activity of the mitochondrial respiratory complexes II, III, and IV. In addition, striatal neuronal cultures expressing $\mathrm{mtH} t \mathrm{tt}$ exhibit decreased expression of respiratory complex $11 .{ }^{7}$ Furthermore, HD patients lose weight despite normal or increased calorie intake and their cortex and basal ganglia have increased lactate levels, indicative of a metabolic defect. $^{8}$ Moreover, mitochondria isolated from the lymphoblasts of HD patients, brain tissue of $\mathrm{mtHtt}$ mice, and $\mathrm{mtH}$ tt-expressing cells all exhibit decreased mitochondrial membrane potential $\left(\Delta \Psi_{\mathrm{m}}\right)$, increased susceptibility to calcium-induced mitochondrial depolarization, and reduced mitochondrial calcium uptake capacity. ${ }^{3,9}$ Finally, mtHtt causes ultrastructural changes of mitochondria and localizes to the mitochondrial outer membrane, ${ }^{10}$ where it can form aggregates. ${ }^{3}$ Incubation of isolated mitochondria with recombinant $\mathrm{mtH}$ tt protein leads to mitochondrial swelling and release of cytochrome $c$, suggesting a direct effect of the protein on mitochondria. ${ }^{10}$

3-Nitropropionic acid (3-NP), an irreversible inhibitor of the mitochondrial respiratory complex II and succinate dehydrogenase, recapitulates HD-like pathology and symptoms in primate and rodent models. ${ }^{11}$ In addition, overexpression of complex II subunits reverts mitochondrial defects and prevents cell death in striatal $\mathrm{mtH}$ tt neurons. ${ }^{7}$ Thus, although it is clear that complex II dysfunction is an important event in HD pathogenesis, ${ }^{2}$ the cause of this dysfunction and how it triggers downstream neuronal cell death is not clear.

In healthy neurons, mitochondria often form long filaments that migrate, fuse, and divide. ${ }^{12}$ The dynamic properties of

\footnotetext{
${ }^{1}$ Apoptosis and Cell Death Program, Burnham Institute for Medical Research, 10901 North Torrey Pines Road, La Jolla, CA 92037, USA and ${ }^{2}$ Burnett School of Biomedical Sciences, College of Medicine, University of Central Florida, 4000 Central Florida Blvd., Orlando, FL 32816, USA

${ }^{*}$ Corresponding author: E Bossy-Wetzel, Biomedical Science Center, University of Central Florida, 4000 Central Florida Blvd., Orlando, FL 32816, USA.

Tel: + 407823 4756; Fax: + 407823 0956; E-mail: ebossywe@ @ail.ucf.edu

Keywords: mitochondria; Huntington's disease; nitric oxide; NMDA receptor; excitotoxicity; reactive oxygen species

Abbreviations: AP5, D-2-amino-5-phosphonopentanoate; DIV, days in vitro; DMEM, Dulbecco's Modified Eagle Medium; HD, Huntington's disease; mtHtt, mutant huntingtin; 3-NP, 3-nitropropionic acid; NAC, $\mathrm{N}$-acetyl-L-cysteine; NMDA, $N$-methyl-D-aspartate; NO, nitric oxide; $\mathrm{O}_{2}^{-}$, superoxide anions; $\mathrm{ONOO}^{-}$, peroxynitrite; $\mathrm{ROI}$, region of interest; RT, room temperature; ROS, reactive oxygen species; $\Delta \Psi_{\mathrm{m}}$, mitochondrial membrane potential; zVAD-fmk, $N$-benzyloxycarbonyl-Val-Ala-Aspfluoromethylketone

Received 04.9.08; revised 03.2.09; accepted 04.2.09; Edited by N Bazan; published online 20.3.09
} 
mitochondria are believed to ensure equal energy and metabolite distribution across long neurites. A filamentous mitochondrial morphology correlates with high bioenergetic functionality and cell survival. Significantly, mutations in mitochondrial fusion factors mitofusin 2 (MFN2) and optic atrophy 1 (OPA1), which result in chronic mitochondrial fission, cause neurodegenerative disorders in humans. ${ }^{12}$ In addition, we recently showed that nitric oxide (NO), $N$-methyl-D-aspartate (NMDA), rotenone, and $\beta$-amyloid peptide, all of which have been implicated in neurodegeneration, evoke excessive mitochondrial fission and neuronal cell death. ${ }^{13,14}$ Finally, we demonstrated that mitochondrial fission occurs in ischemic stroke in vivo and is required for NO-mediated neuronal demise. ${ }^{13}$ Thus, the importance of mitochondrial fragmentation in neurodegeneration is becoming increasingly apparent and its potential role in HD-associated mitochondrial dysfunction is an important area for investigation.

As respiratory complex II inhibition is functionally implicated in HD pathogenesis in humans and animal models, $2,3,7,8,10,15$ we studied here the mechanisms underlying 3-NP-mediated neuronal injury using time-lapse fluorescence imaging. We used quantitative time-lapse fluorescence imaging to dissect the sequence of downstream events in response to complex II inhibition. We show that 3-NP evokes two distinct signals on mitochondria. The first signal is a direct effect of 3-NP on mitochondrial bioenergetics, causing a dramatic and rapid ATP drop and a mild reactive oxygen species (ROS) increase, without alteration in mitochondrial morphology and neuronal survival. The second signal, following several hours later with variable onset, is characterized by activation of glutamate receptors of the NMDA subtype, which in turn causes a second ROS rise and mitochondrial fragmentation. Importantly, this secondary event triggers neuronal cell death. Our results indicate that secondary excitotoxicity, rather than the initial 3-NP-induced bioenergetic deficits characterized by ATP drop and mild ROS increase, causes mitochondrial fission and neuronal cell death. These findings provide a mechanistic link between mitochondrial dysfunction and excitotoxicity in a cellular model relevant to HD. They also have important implications for the development of new markers and therapies to combat $\mathrm{HD}$ and other neurodegenerative disorders.

\section{Results}

3-NP induces changes in mitochondrial morphology. Mitochondrial dysfunction plays a pivotal role in HD pathogenesis. Recent reports indicate that mitochondria undergo dramatic fragmentation and movement arrest in response to various neurotoxic insults. ${ }^{13,14,16-18}$ To determine the effect of 3-NP on mitochondrial morphology, we investigated the mitochondrial morphology of pure rat cortical neurons expressing DsRed2-Mito in response to 3-NP $(10 \mathrm{mM})$ by $3 \mathrm{D}$ fluorescence wide-field microscopy. Mitochondria, which exhibit long and tubular morphology in healthy control neurons, became dramatically shorter and rounder in response to 3-NP (Figure 1A).

To follow, more precisely, the sequence of mitochondrial morphology changes over time, we tracked DsRed2-Mito- expressing neurons by 3D time-lapse fluorescence microscopy after 3-NP (10 mM) exposure for several hours. Six to twelve hours after 3-NP exposure, mitochondria were converted from elongated and filamentous to punctate (Figure 1B). We were able to differentiate two types of morphological change. First, a subpopulation of contracting mitochondria, which reduced their length (condensed), but did not increase in number (Figure 1Ba). Second, a subpopulation that underwent fragmentation (fission) into several smaller sized structures with an increase in number (Figure 1Bb). Both mitochondrial condensation and fragmentation occurred in a single neuron following 3-NP exposure. We used the term 'punctate mitochondria' to describe both of these 3-NP-mediated changes in mitochondrial morphology.

3-NP causes a rapid decrease in ATP levels that precedes changes in mitochondrial morphology. As 3-NP is an irreversible inhibitor of succinate dehydrogenase and blocks respiration, we asked whether it affects intracellular ATP concentration. To evaluate changes in ATP concentration and correlate them with changes in mitochondrial morphology, we exposed neurons to increasing concentrations of 3-NP $(0.1-10 \mathrm{mM})$ and measured ATP levels in cell lysates 1,3 , and $6 \mathrm{~h}$ later. Treated neurons showed a rapid dose- and time-dependent ATP decrease. Specifically, as early as $1 \mathrm{~h}$ after 3-NP treatment, ATP levels decreased by $32 \%$ at $1 \mathrm{mM} 3-\mathrm{NP}$ and by $62 \%$ at $10 \mathrm{mM} 3-\mathrm{NP}$ (Figure 2A). This drop was even more severe at $3 \mathrm{~h}(40 \%$ at $1 \mathrm{mM} 3-\mathrm{NP}$ and $75 \%$ at $10 \mathrm{mM}$ 3-NP; Figure 2A) and became more dramatic after $6 \mathrm{~h}$, with a maximal drop of $22 \%$ at $0.1 \mathrm{mM} 3-\mathrm{NP}$ and $79 \%$ at $10 \mathrm{mM}$ 3-NP (Figure 2A). The raw data of the ATP measurements are shown in Supplementary Figure 1.

To explore the relationship between ATP decline and changes in mitochondrial morphology, we analyzed mitochondrial morphology after exposing DsRed2-Mito-expressing neurons to increasing 3-NP concentrations $(0.1-10 \mathrm{mM})$. We fixed neuronal cultures at 1,3 , and $6 \mathrm{~h}$ after $3-\mathrm{NP}$ exposure and analyzed mitochondrial morphology using fluorescence microscopy. Unlike the early decline in ATP production, we did not observe any significant increase in the number of neurons with punctate mitochondria at any 3-NP concentration in the first hour (Figure $2 \mathrm{Aa}$ and $\mathrm{Ba}$ ). However, after $3 \mathrm{~h}$, a significant increase in the number of such neurons was apparent at 5 or $10 \mathrm{mM}$ 3-NP (Figure 2Ab and $\mathrm{Bb}$ ). This increase persisted at $6 \mathrm{~h}$ at 5 and $10 \mathrm{mM} 3-\mathrm{NP}$ and the number of neurons exhibiting punctate mitochondria increased to 65 and $95 \%$, respectively (Figure $2 A c$ and Bc). At $6 \mathrm{~h}$, the baseline of punctate mitochondrial morphology was increased. This could be due to variability in the neuronal cultures.

In sum, although 3-NP caused a decrease in ATP even at low levels and within the first hour of exposure, mitochondrial morphology changes only occurred at the highest levels of 3-NP, and then only at the later time points. Thus, ATP decline greatly precedes changes in mitochondrial morphology. Finally, despite the profound ATP drop and subsequent mitochondrial morphology changes in response to 3-NP 
A
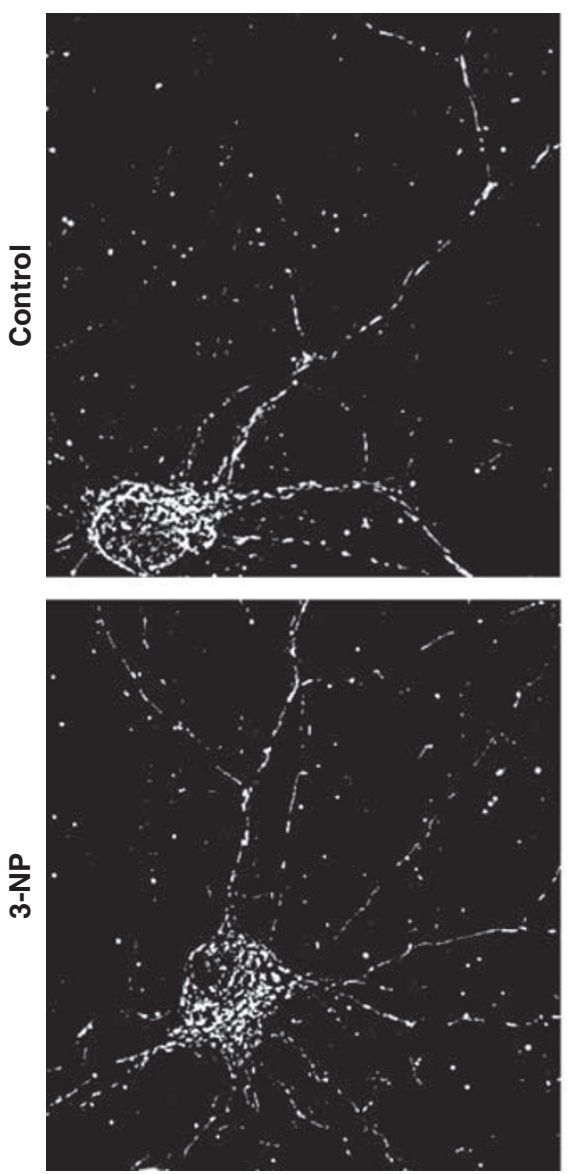

$6 h$
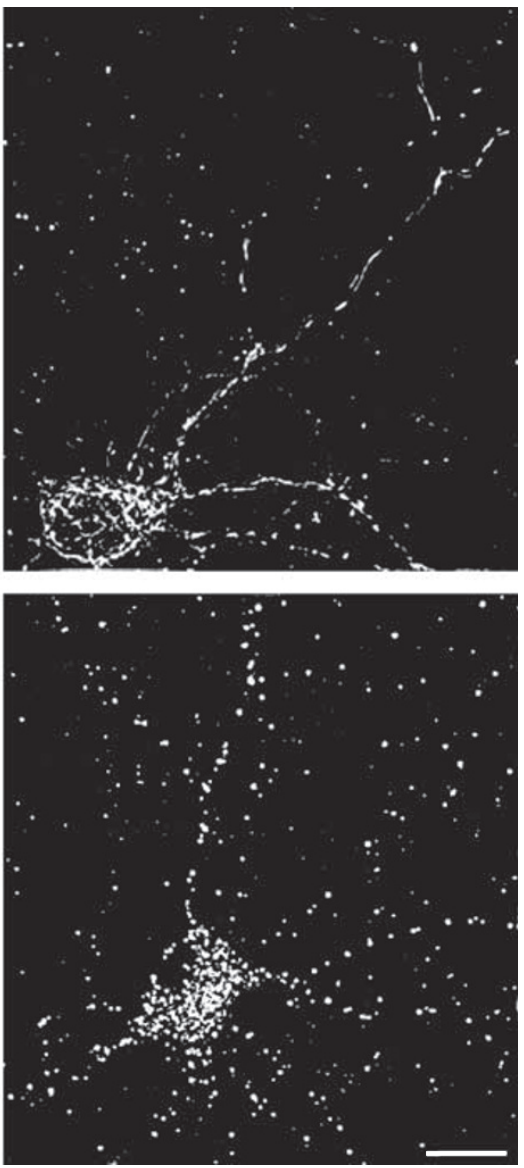

B

3-NP
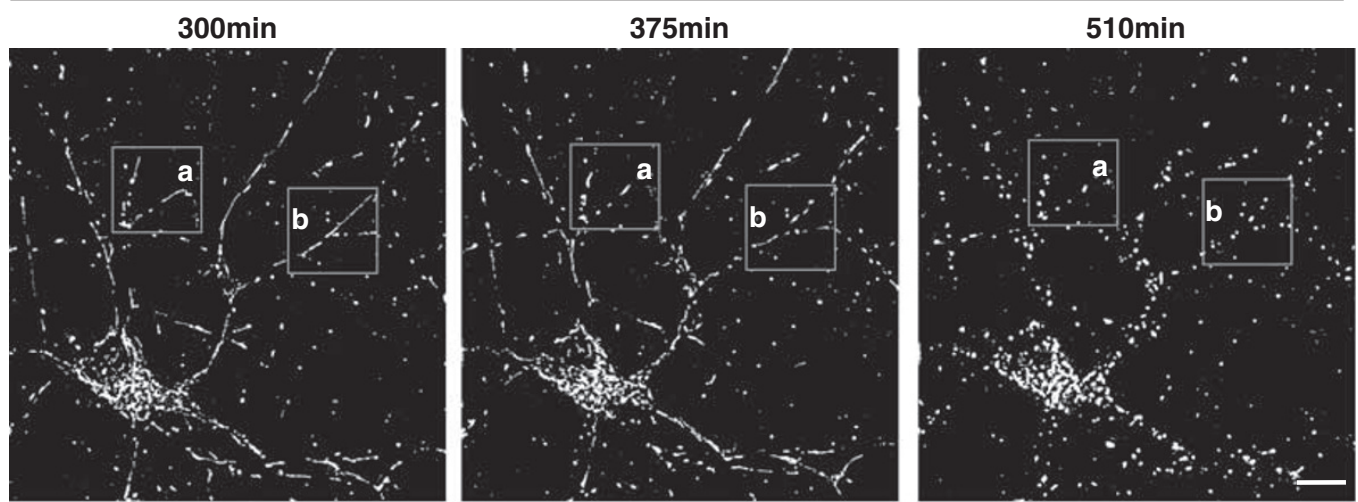

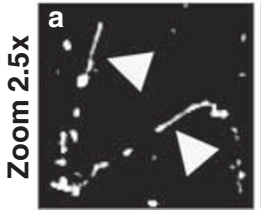

$300 \mathrm{~min}$

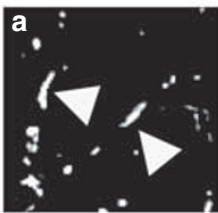

$375 \mathrm{~min}$

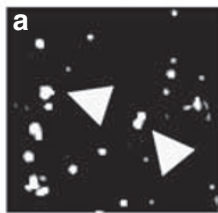

$510 \mathrm{~min}$

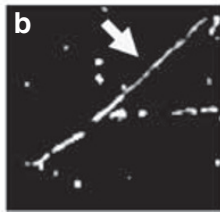

$300 \mathrm{~min}$

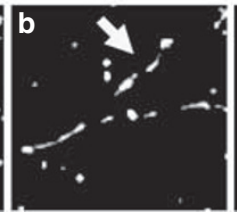

375min

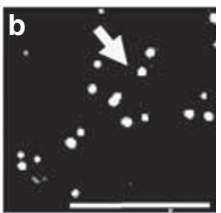

$510 \mathrm{~min}$

Figure 13 3-NP induces punctate mitochondrial morphology. DsRed2-Mito expressing purified cortical neurons were exposed to $3-\mathrm{NP}$ (10 mM) at $12 \mathrm{DIV}$ and changes in mitochondrial morphology were visualized using wide-field fluorescence 3D time-lapse imaging. (A) Representative frames of the time-lapse series of a control neuron (upper panel) and 3-NP-exposed neuron (lower panel) at time zero and after $6 \mathrm{~h}$. Mitochondrial morphology of the control neuron remains unaltered during this time period (upper panel). In contrast, the 3-NP-exposed neuron (lower panel) exhibits a change from tubular to punctate mitochondrial morphology. Scale bar, $10 \mu \mathrm{m}$. (B) Representative frames of a 3D timelapse series featuring a DsRed2-Mito expressing cortical neuron at $300 \mathrm{~min}, 375 \mathrm{~min}$ and $510 \mathrm{~min}$ after 3-NP exposure. (a and $\mathbf{b}$, lower panels) show a 2.5x zoom of the region of interest (ROI), marked within the white square. Mitochondria show both condensation (a, white arrowheads) as well as fragmentation (b, white arrows). Scale bar, $10 \mu \mathrm{m}$ 

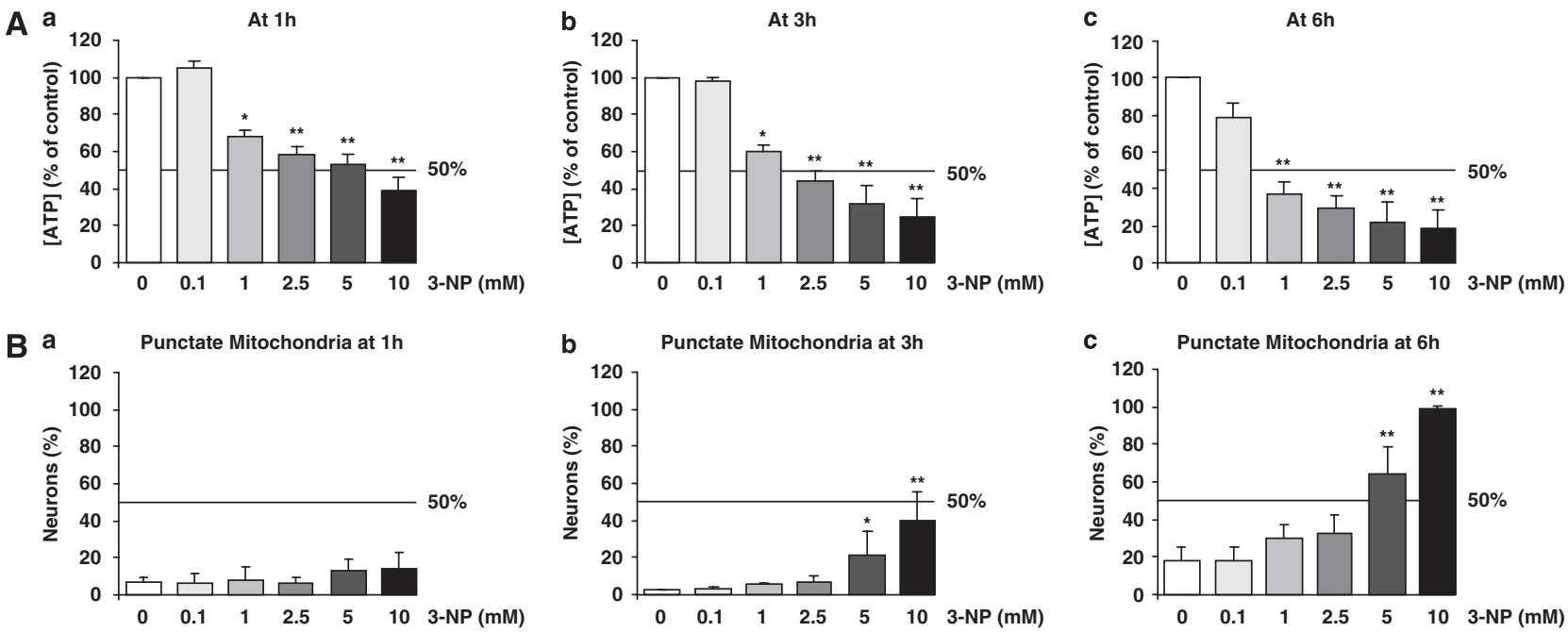

Figure 2 3-NP induces an instant and dose-dependent decline in ATP, preceding appearance of punctate mitochondria. Cortical neurons were exposed to increasing 3 $\mathrm{NP}$ concentrations $(0,0.1,1,2.5,5,10 \mathrm{mM})$, and ATP concentration and mitochondrial morphology were monitored after 1,3 , and $6 \mathrm{~h}$. (A) 3-NP induces a significant decrease in neuronal ATP concentration (measured as percentage of control) starting at $1 \mathrm{mM} \mathrm{NP}-3$ and at $1 \mathrm{~h}$, which further declines at 2.5, 5, and $10 \mathrm{mM}$ 3-NP and increasing time. (B) DsRed2-Mito expressing cortical neurons were exposed to increasing 3-NP concentrations. After cell fixation, the percentage of neurons with punctate mitochondria was scored by fluorescence microcopy. Only at 3 and $6 \mathrm{~h}$ was a significant increase in mitochondrial fragmentation apparent at 5 and $10 \mathrm{mM} 3-\mathrm{NP}$. Bar graph represent the mean of three independent experiments and error bars indicate \pm S.E.M. Significant differences are indicated by symbols ${ }^{*}(P<0.05)$ and ${ }^{* *}(P<0.01$; one-way ANOVA and Fisher's $t$-test $)$

(Figure 2), we detected no signs of cell death over the 6-h time frame (data not shown).

3-NP evokes a biphasic ROS increase. Mitochondria are the primary sources of ROS. To analyze the link between bioenergetic defects such as ROS production and mitochondrial morphology changes, we utilized a vector encoding reduction-oxidation sensitive GFP (ro-GFP) ${ }^{19}$ targeted to mitochondria via a pyruvate dehydrogenase E1 $\alpha$ sequence (ro-GFP-Mito). This novel tool allows us to monitor changes in mitochondrial dynamics and morphology and ROS production simultaneously in real time. Ro-GFPMito has excitation wavelengths at $403 \mathrm{~nm}$ (oxidized) and $490 \mathrm{~nm}$ (reduced); thus, an increased 403/490 ratio reflects increased ROS production. We transfected cortical neurons with the ro-GFP-Mito vector and monitored both 403/490 ratios and mitochondrial morphology simultaneously by quantitative 3D time-lapse fluorescence microscopy (in each experiment, we chose 10-20 neurons per condition). Figure $3 \mathrm{~A}$ shows representative images of vehicle-treated control (EBSS buffer) (a-f) and 3-NP-exposed $\left(a^{\prime}-f^{\prime}\right)$ neurons over a time period of $12 \mathrm{~h}$. Control neurons exhibit typical, tubular mitochondria and their average 403/490 ratio remained unaltered during the imaging period (Figure $3 \mathrm{Aa}-\mathrm{f}$ and corresponding enlargements below). In contrast, 3-NPexposed neurons exhibited an instant increase in the 403/ 490 ratio in the first hour in the majority of neurons (Figure $3 \mathrm{Aa}^{\prime}-\mathrm{c}^{\prime}$ and corresponding enlargements). Respiratory complex II inhibition by 3-NP likely caused this increase in ROS. Unexpectedly, this initial rise in ROS persisted in most neurons and was followed, after a variable lag period lasting several hours in most cases, by a second dramatic increase in ROS, which was accompanied by a conversion from tubular to punctate mitochondrial morphology (Figure $3 A d^{\prime}-f^{\prime}$ and corresponding enlargements).

Figure 3B collectively shows the $403 / 490$ ratios of several control $(n=15)$ and 3-NP-treated $(n=14)$ neurons. The $403 / 490$ ratio did not change in control neurons over the $12 \mathrm{~h}$ period except for two neurons (gray and light pink trace). The thick red lines in Figure 3B correspond to the control and 3-NP exposed neuron depicted in Figure 3A. However, several (9 out of 14) 3-NP (10 mM)-exposed neurons exhibit biphasic increases in the $403 / 490$ ratio - the first occurring in the first hour after 3-NP treatment (13 out of 14 neurons) and the second beginning after a variable lag phase - suggestive of two distinct signals (Figure 3B). Four neurons exhibited an early second peak (between 100 and 270 min; pink, red, light blue, and orange trace) and five neurons showed a late second peak (between 400 and $700 \mathrm{~min}$; green, bold red, black, light purple, and purple trace). These data demonstrate that 3-NP treatment causes a biphasic ROS increase. Importantly, only the second ROS peak correlates with mitochondrial fragmentation and shortening. Thus, 3-NPinduced respiratory inhibition and a drop in ATP does not cause mitochondrial fragmentation, but rather a secondary signal, which coincides with a second rise in ROS, causes the changes in mitochondrial morphology.

Antioxidants block 3-NP-induced mitochondrial morphology changes. Next, we asked if ROS was responsible for the observed occurrence of punctate mitochondria. To test this, we pretreated neuronal cultures expressing DsRed2-Mito with the widely used thiol antioxidant $\mathrm{N}$-AcetylL-cysteine (NAC), which powerfully blocks nitrosative and oxidative stress, and assessed mitochondrial morphology by fluorescence microscopy in the presence or absence of 3-NP $(10 \mathrm{mM})$. Figure 4 shows representative dendritic processes 
A
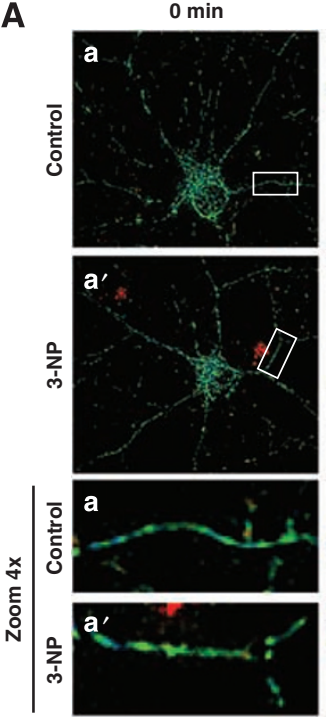

B

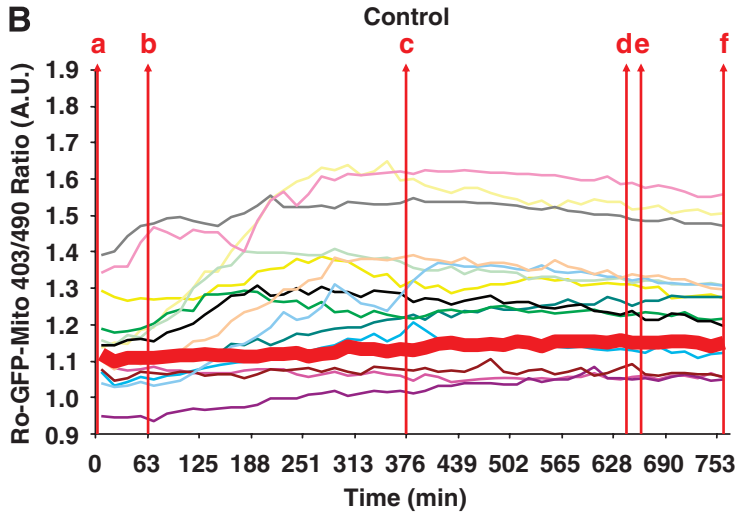

$63 \mathrm{~min}$
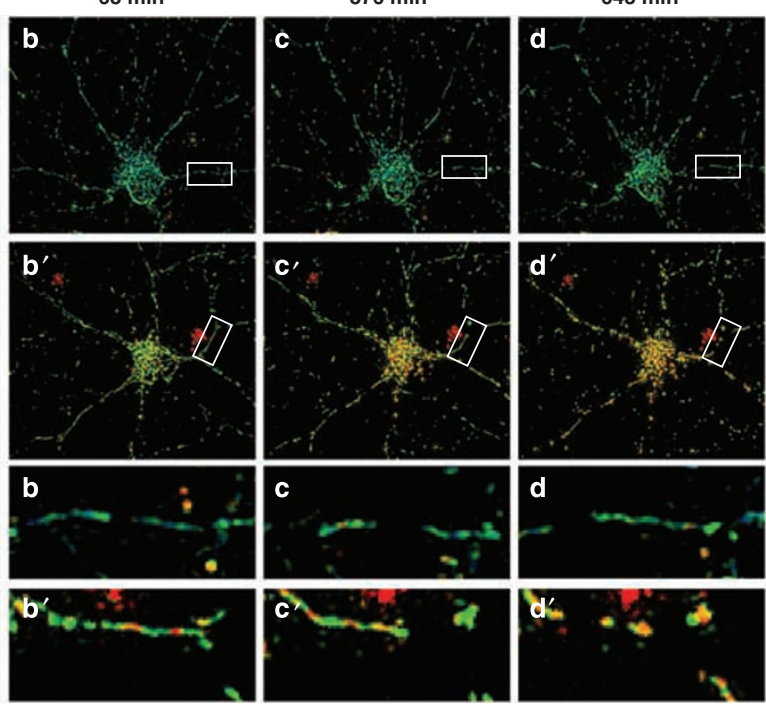

$659 \min$
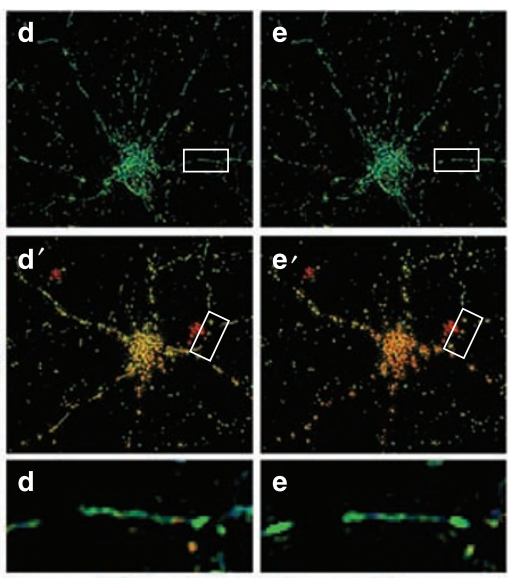

$753 \mathrm{~min}$

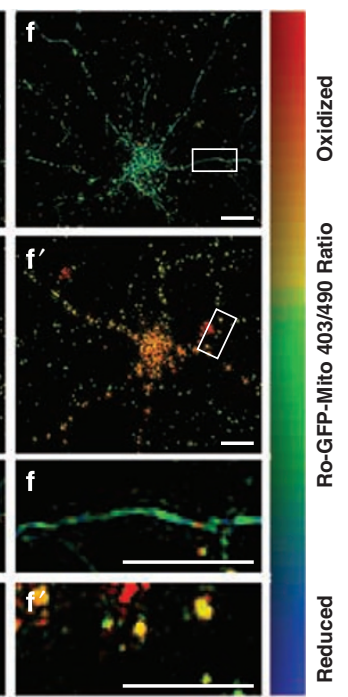

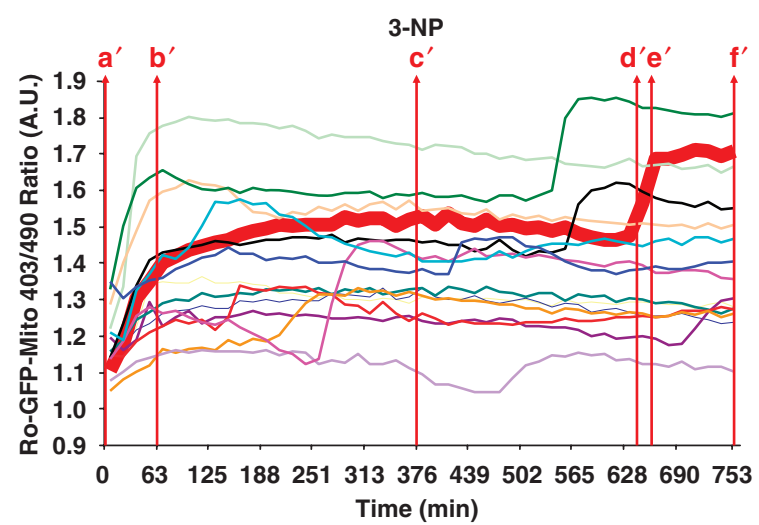

Figure 3 3-NP induces a biphasic ROS increase, and mitochondrial fragmentation accompanies only the second ROS peak. Cortical neurons were transfected with the roGFP-Mito vector and mitochondrial ROS and morphology were monitored simultaneously be cyclic revisiting of $\sim 15$ neurons using 3D quantitative fluorescence wide-field microscopy. (A) Representative frames of a 3D time-lapse series of one control neuron (upper panels) and one 3-NP-exposed neuron. The change in the ratio $403 / 490 \mathrm{~nm}$ and oxidation was visualized by applying pseudocolor. Ro-GFP-Mito oxidation is recognized by a shift from cold to warmer colors. At 63 min, 3-NP causes an increase in ro-GFPMito oxidation, but no change in mitochondrial morphology. At $643 \mathrm{~min}$, a second increase in ro-GFP-Mito oxidation occurs, which coincided with mitochondrial fragmentation. The bottom two rows depict a $\times 4$ zoom of the rectangular marked ROI. Scale bars, $10 \mu \mathrm{m}$. (B) Traces show the kinetics of $403 / 490 \mathrm{~nm}$ ratio changes in arbitrary units (AU) of all control (left) and 3-NP-treated (right) neurons evaluated in (A). Each colored line represents a single neuron and the thick red line corresponds to the control or 3-NP exposed neuron depicted in (A). The control neuron does not show changes in the 403/490 nm ratio (left), whereas the 3-NP-exposed neuron shows two increases (B, right), the first at $63 \mathrm{~min}$ and the second at $643-659 \mathrm{~min}$. In (B), red vertical lines $(\mathbf{a}-\mathbf{f})$ and $\left(\mathbf{a}^{\prime}-\mathbf{f}^{\prime}\right)$ correspond to the time points imaged in $(\mathbf{A})$

of these neurons at $12 \mathrm{~h}$. At $12 \mathrm{~h}$, control neurons (a) or neurons pretreated for $3 \mathrm{~h}$ with $50 \mu \mathrm{M}$ NAC (b) exhibited tubular mitochondria, whereas neurons treated with 3-NP exhibited only punctate mitochondria (c). In contrast, neurons pretreated with NAC $(50 \mu \mathrm{M}, 3 \mathrm{~h})$ and then exposed to 3-NP $(10 \mathrm{mM})$ exhibited tubular mitochondria (d). Quantification of these effects showed that $90.4 \%$ of neurons exposed to 3-NP exhibited punctate mitochondria (Figure 4e), which significantly decreased to $49.9 \%$ following NAC pretreatment. Only 21.1 and $21.4 \%$ of control and NAC-pretreated neurons, respectively, exhibited punctate mitochondrial morphology. These observations demonstrate that 3-NP-induced transition from a tubular to punctate mitochondrial morphology depends, in part, on oxidative and nitrosative stress, consistent with our and others previous observations. ${ }^{13,14}$
NMDA receptor activity is responsible for 3-NP-induced punctate mitochondrial morphology. Excitotoxicity mediated by glutamate receptors of the NMDA subtype has been implicated in HD and 3-NP neuronal toxicity, however the relationship to mitochondrial morphology changes is not clear. $^{20-26}$ Therefore, we tested the hypothesis that NMDA receptor activation causes 3-NP-induced punctate mitochondrial morphology. DsRed2-Mito-expressing neurons were exposed to 3-NP (10 mM) alone, to D-2-amino-5-phosphonopentanoate (AP5; $100 \mu \mathrm{M}$ ) alone, or a combination of both for $12 \mathrm{~h}$ (Figure 5). AP5 is a NMDA receptor antagonist. As anticipated, all neurons initially exhibited tubular mitochondria, and after $12 \mathrm{~h}$ of $3-\mathrm{NP}$ exposure, most neurons exhibited punctate mitochondria (Figure $5 \mathrm{a}$ and b). However, neurons treated with both 3-NP and AP5 (Figure $5 \mathrm{c}$ ) 

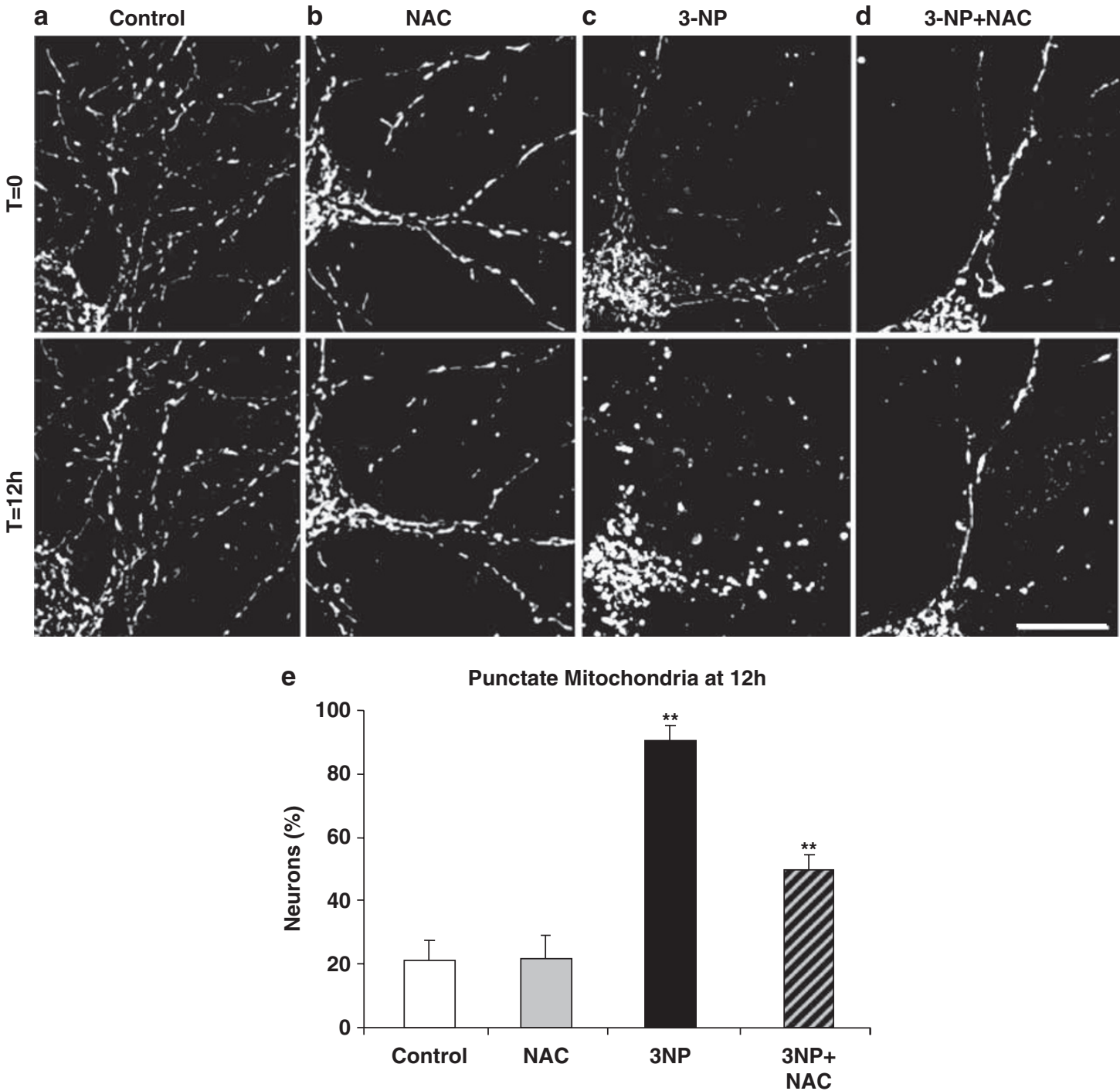

Figure 4 Oxidative and nitrosative stress inhibition alleviates 3-NP-induced formation of punctate mitochondria. Representative snap shots of a time-lapse series of a DsRed2-Mito-transfected control neuron (a), a 3-NP $(10 \mathrm{mM})$ exposed neuron $(\mathbf{b})$, a neuron pretreated $3 \mathrm{~h}$ with NAC $(50 \mu \mathrm{M})$ followed by 3-NP (10 mM) exposure (c), and a neuron pretreated with NAC alone $(50 \mu \mathrm{M})$ (d) - all at time zero ( $T=0$, upper) and $12 \mathrm{~h}$ later $(T=12 \mathrm{~h}$, lower). Scale bar, $10 \mu \mathrm{m}$. (e) Bar graph of the percentage of neurons with punctate mitochondria at $12 \mathrm{~h}$ of 3-NP exposure. Data represent four independent experiments and error bars indicate \pm S.E.M. Significant differences are indicated by symbols $^{* *}(P<0.01$; one-way ANOVA followed by Fisher's $t$-test $)$

exhibited mostly tubular mitochondria, similar to control (Figure 5a) and AP5-treated (Figure 5d) neurons.

Quantification of these findings showed that $88.1 \%$ of the neurons in cultures treated with 3-NP exhibited punctate mitochondria. However, the presence of the AP5 significantly reduced 3-NP-mediated increases in punctate mitochondrial morphology to $34.2 \%$. Only 20.6 and $21.3 \%$ of control neurons and neurons treated with AP5 alone, respectively, exhibited punctate mitochondria (Figure 5e). Similarly, when we evaluated cell death by chromatin condensation and soma shrinkage, we found that AP5 cotreatment with 3-NP mitigated 3-NP-induced neuronal cell death (Figure 5f). The 3-NP-induced cell death lacks caspase-3 enzyme activation (Supplementary Figure 2) and thus may proceed by a nonapoptotic pathway. Overall, these data indicate that downstream excitotoxic signals mediated by NMDA receptors cause 3-NP-induced punctate mitochondrial morphology and neuronal cell death.

AP5 blocks the second 3-NP-induced ROS peak. 3-NP treatment of neurons elicits a biphasic ROS increase (Figure 3). We therefore speculated that NMDA receptor activation might cause the second ROS peak. To test this hypothesis, we treated neurons expressing ro-GFP-Mito with vehicle (Figure 6a), 3-NP (10 mM) alone (Figure 6b), AP5 alone (Figure 6c), or 3-NP plus $100 \mu \mathrm{M}$ AP5 (Figure $6 \mathrm{~d}$ ), and monitored the $403 / 490$ ratio by quantitative $3 D$ time-lapse fluorescence microscopy of approximately 15 neurons for each condition by cyclic revisitation over a period of $12 \mathrm{~h}$. Control neurons (Figure 6a) and neurons treated with AP5 alone (Figure $6 \mathrm{c}$ ) did not exhibit significant changes in ROS generation or mitochondrial morphology. Again, 3-NP 
a
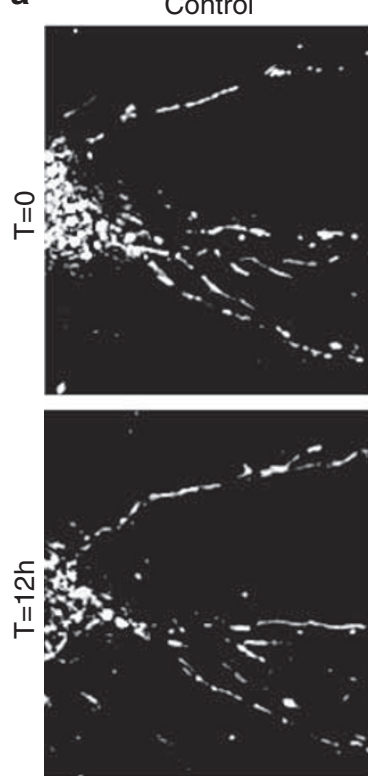

e

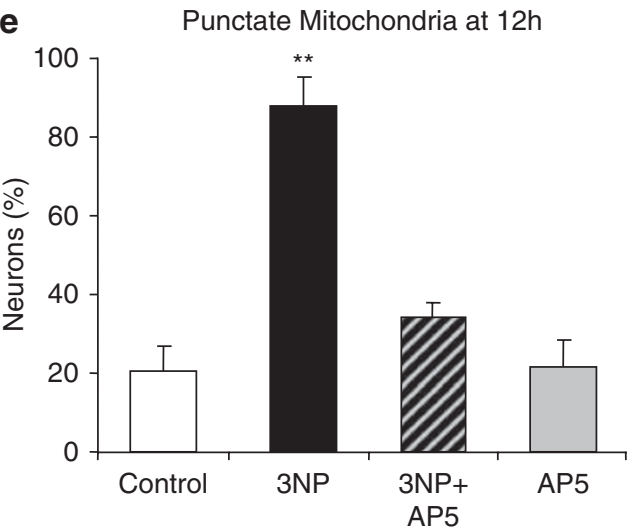

b $\quad 3-N P$
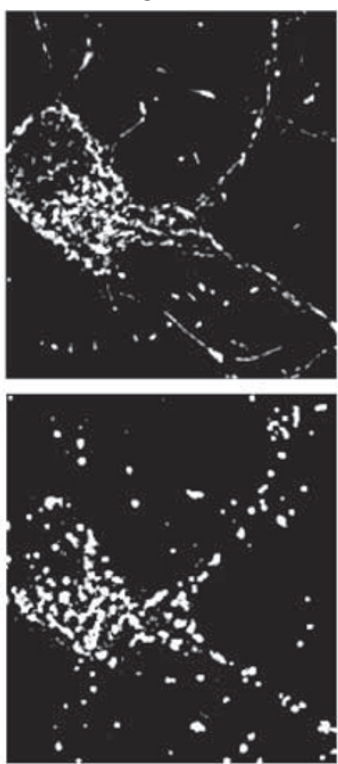

c $\quad 3-\mathrm{NP}+\mathrm{AP} 5$
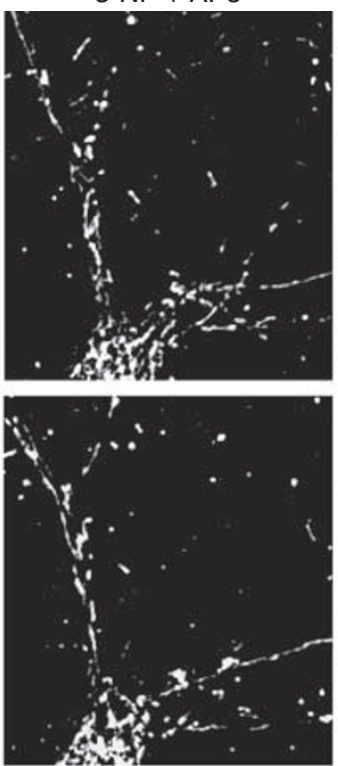

d
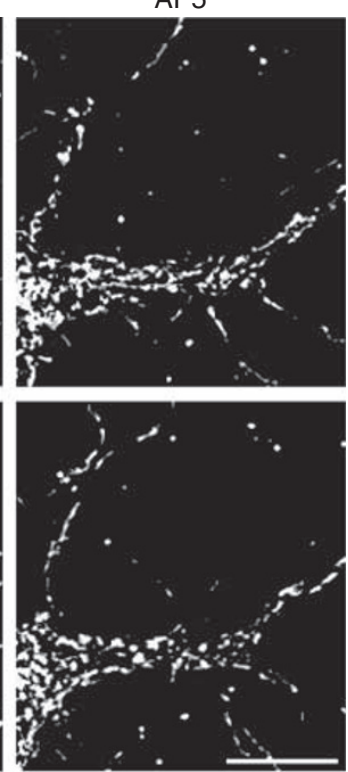

f

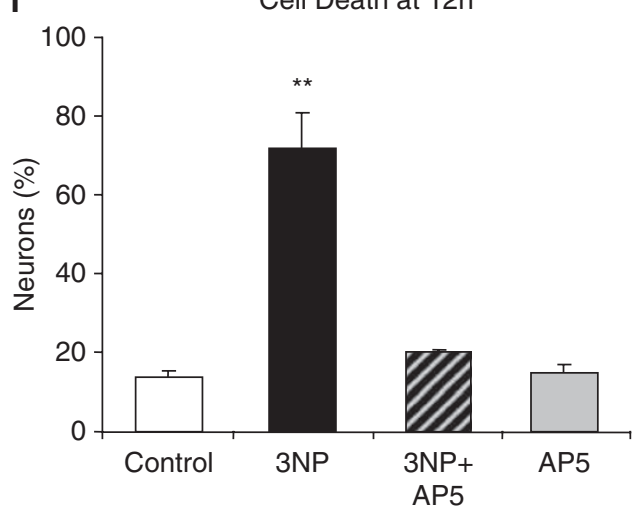

Figure 5 NMDA receptor antagonists abolish the 3-NP-induced formation of punctate mitochondria. Representative images of a DsRed2-Mito transfected control neuron (a), a neuron treated with 3-NP (10 mM; b), a neuron treated with AP5 $(100 \mu \mathrm{M})$ plus 3-NP $(10 \mathrm{mM} ; \mathbf{c})$, and a neuron treated with AP5 alone (100 $\mu \mathrm{M}$; d), all at the beginning of treatment $(T=0$, upper) and $12 \mathrm{~h}$ later $(T=12 \mathrm{~h}$, lower). Scale bar, $10 \mu \mathrm{m}$. (e) Bar graph shows the percentage of neurons with punctate mitochondrial morphology at $12 \mathrm{~h}$ of 3-NP exposure. (f) Bar graph of the percentage of cell death measured by chromatin condensation, shrunken soma, and fragmented mitochondria at $12 \mathrm{~h}$ after $3-\mathrm{NP}$ exposure. Data represent 3-4 independent experiments, and error bars indicate \pm S.E.M. Significant differences are indicated by symbols ${ }^{* *}(P<0.01$; one-way ANOVA followed by Fisher's t-test)

exposure induced a biphasic ROS increase (9 out of 15 neurons; Figure 6b). Remarkably, AP5 had no impact on the first ROS peak, but dramatically abolished the second peak in ROS production (13 out of 14 neurons; Figure $6 \mathrm{c}$ ). These data suggest that NMDA receptor activation causes the second ROS increase, the one that corresponds with a conversion to punctate mitochondrial morphology and neuronal cell death (Figure 5).

\section{Discussion}

Chronic decrease in mitochondrial fusion or increase in mitochondrial fission plays a critical role in cell death and neurodegeneration. ${ }^{12}$ Here, we examined the consequences of complex II inhibition by 3-NP with quantitative 3D fluorescence time-lapse microscopy. Using this technique, we established the temporal and spatial relationship between respiratory inhibition, energy deficits, ROS production, mitochondrial fission, and neuronal cell death. We found that 3-NP evokes an immediate ATP drop in cortical neurons that correlates with a mild but sustained ROS increase at $30 \mathrm{~min}$ after 3-NP exposure. Our data are consistent with previous reports showing a rapid ATP drop on 3-NP exposure, which is accompanied by an increase in ROS. ${ }^{27-29}$ This initial wave in ROS does not cause mitochondrial fragmentation or cell death. However, an unexpected and sporadic secondary phase of ROS increase followed this primary phase. The onset of this delayed, secondary ROS increase varied greatly, ranging from one to several hours, and occurred only in a subset of neurons, suggesting that it was caused by a second intracellular signal that is activated in response to the initial energy drop and changes in the redox milieu. Remarkably, 
a

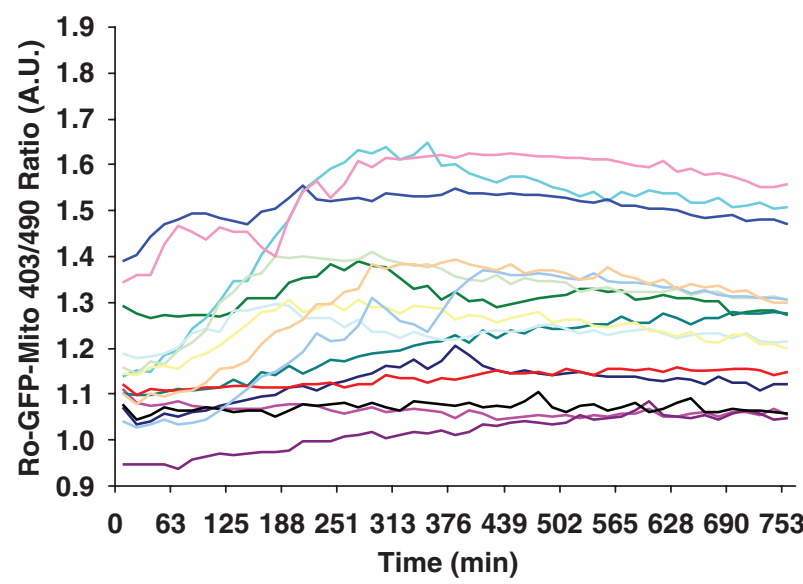

C

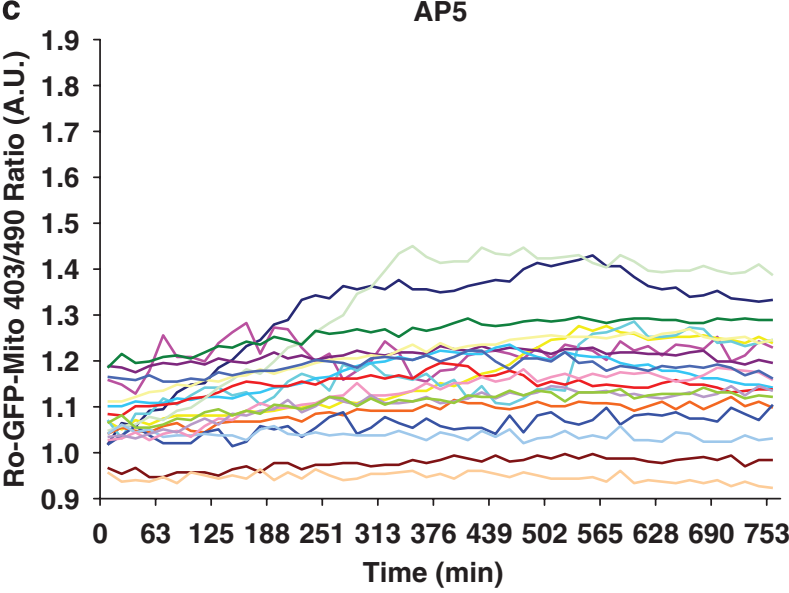

b
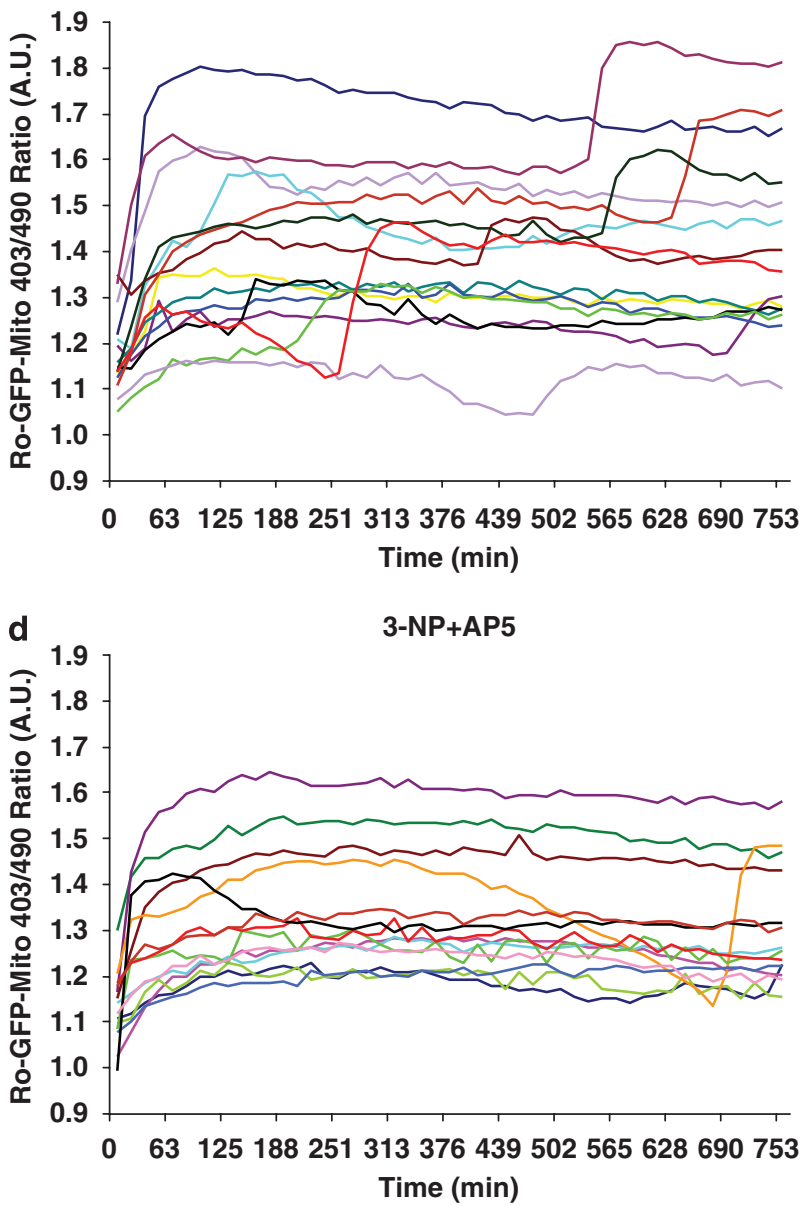

Figure 6 NMDA receptor antagonists abolish 3-NP-induced second ROS peak. Ro-GFP-expressing neurons were treated with 3-NP and AP5 alone or a combination of 3-NP plus AP5 and $\sim 15$ neurons were subjected to quantitative fluorescence time-lapse microscopy over a 12-h time period. Each trace represents the ro-GFP Mito 403/490 ratio in arbitrary units of a single neuron over time of vehicle treated control neurons (a), neurons treated with 3-NP (10 mM; b), neurons treated with AP5 (100 $\mu \mathrm{M} ; \mathbf{c})$, and neurons treated with 3-NP plus AP-5 $(100 \mu \mathrm{M} ; \mathbf{d})$

although the mitochondrial morphology remained tubular and unaltered during the first rise in ROS, the second ROS peak correlated with profound mitochondrial fragmentation. NAC, a free radical scavenger, blocked the 3-NP-induced mitochondrial fragmentation, suggesting that a subspecies of free radicals, such as $\mathrm{NO}$ and peroxynitrite $\left(\mathrm{ONOO}^{-}\right)$, may evoke the filamentous to punctate mitochondrial morphology change. Most importantly, glutamate receptor antagonist AP5 blocked the second ROS peak, mitochondrial fragmentation, and neuronal cell death. Taken together, our data provide additional evidence that secondary excitotoxicity may play a role in HD-linked neuronal injury caused by complex II inhibition. ${ }^{20-26}$ Additionally, our results place mitochondrial fragmentation downstream of glutamate receptor activation, but upstream of ROS production and neuronal cell death. A model summarizing our results is shown in Figure 7.

There is strong evidence that excitotoxic pathways play a central role in $\mathrm{HD}$ and other neurodegenerative disorders. ${ }^{20-26,30}$ Medium spiny neurons and cortical neurons, which are particularly affected in HD, receive glutamatergic inputs. Thus, overactivation of NMDA receptors may lead to neuronal cell death by excitotoxicity, providing an explanation for their increased vulnerability in HD. Indeed, transgenic $\mathrm{mtH}$ tt mice show increased NMDA receptor activity in neurons. ${ }^{30}$ Astrocytes and ATP-dependent glutamate aspartate transporters mediate the clearance of extracellular glutamate. $\mathrm{mtH} t \mathrm{tt}$ reduces the expression of GLT-1 in transgenic mice. ${ }^{31}$ In addition, expression of $\mathrm{mtH} t \mathrm{tt}$ in glial cells increases neuronal excitotoxicity. ${ }^{32}$ A drop in ATP, here elicited by $3-N P$, may cause reduced glutamate reuptake by ATP-dependent transporters. Alternatively, energy failure may cause a gradual plasma membrane potential decline due to decreased activity of ATP-dependent ion pumps and channels.

Recent reports indicate that $\mathrm{mtHtt}$ impairs mitochondrial function and energy production by directly interacting with the organelle or modulating transcription of nuclear encoded mitochondrial proteins. ${ }^{3,10,33}$ In both events, the primary energy impairment and ATP drop may lead to decline of $\mathrm{Na}^{+} / \mathrm{K}^{+}$ATPases and plasma membrane depolarization, 


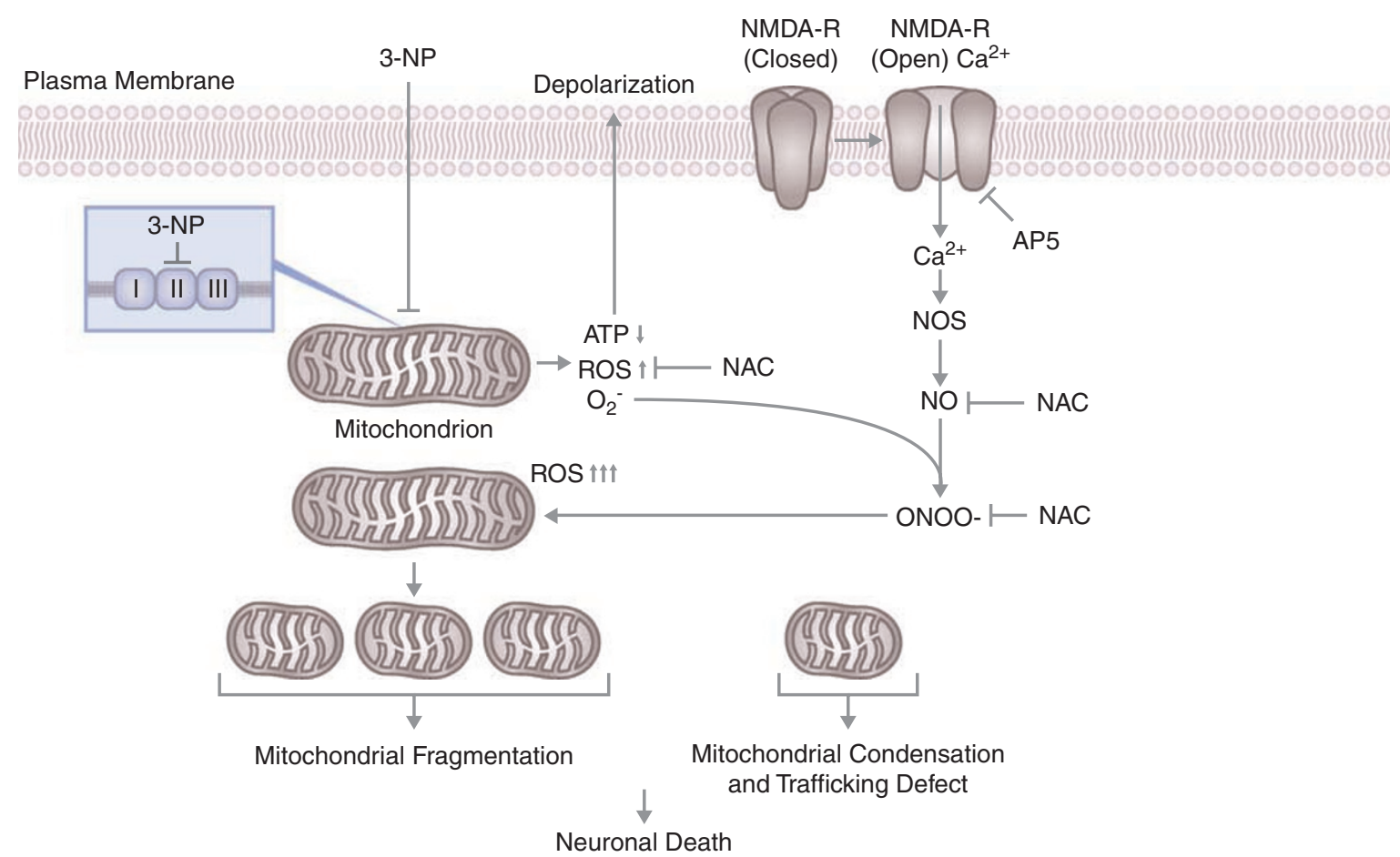

Figure 7 Model of NMDA- and oxidative/nitrosative stress-mediated mitochondrial fragmentation. 3-NP induces mitochondrial respiratory complex II inhibition, which in turn triggers a rapid ATP drop and increase in ROS. The energy deficit may lead to plasma membrane depolarization, which may release the $\mathrm{Mg}^{2+}$ block of voltage-gated NMDA receptors (NMDA-R). Opening of NMDA-R causes intracellular $\mathrm{Ca}^{2+}$ influx, which in turn leads to nitric oxide synthase (NOS) activation and generation of nitric oxide (NO). NO can combine with $\mathrm{O}^{2-}$ to form $\mathrm{ONOO}^{-}$. This additional increase in free radicals, in particular $\mathrm{ONOO}^{-}$can result in mitochondrial fragmentation and trafficking defects, which eventually triggers neuronal cell death. Blocking NMDA-R with AP5 and ROS with NAC blocks mitochondrial fragmentation and neuronal cell death

which in turn releases the voltage-sensitive $\mathrm{Mg}^{2+}$ block of NMDA receptors, causing $\mathrm{Ca}^{2+}$ and $\mathrm{Na}^{+}$influx, NO synthase (NOS) activation, and NO production. NO can combine with superoxide anions $\left(\mathrm{O}_{2}^{-}\right)$to form neurotoxic radical $\mathrm{ONOO}^{-}$.

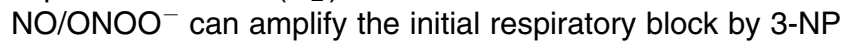
by irreversible inhibition of respiratory complexes. Furthermore, $\mathrm{NO} / \mathrm{ONOO}^{-}$can activate mitochondrial fragmentation and expression of MFN or the dominant-negative mutant of dynamin-related protein $1\left(\mathrm{DRP} 1^{\mathrm{K} 38 \mathrm{~A}}\right)$ blocks this event and increases neuronal survival. ${ }^{13}$ Thus, it is conceivable that the second rise in free radicals in response to 3-NP is due to $\mathrm{NO} / \mathrm{ONOO}^{-}$, which in turn triggers mitochondrial fragmentation and neuronal cell death. Supporting this possibility, Slack and colleagues reported that oxidative stress induces mitochondrial fission before neuronal cell death, and blocking this event by MFN2 expression is neuroprotective. ${ }^{17}$ Whether components of the mitochondrial fission and fusion machinery are directly regulated by nitrosative/oxidative stress remains a question for future investigation. That dynamin, a GTPase related to the mitochondrial fission factor DRP1 that regulates endocytosis, is S-nitrosylated suggest that this might be the case. S-nitrosylation increases dynamin self-assembly, GTPase activity, and membrane targeting. ${ }^{34}$ In sum, NMDA receptor activation may cause a positive feed-back loop that exacerbates the initial bioenergetic defect initiated by 3-NP and subsequently triggers $\mathrm{NO} / \mathrm{ONOO}^{-}$production, mitochondrial fragmentation, and neuronal cell death. Of note is that the
3-NP-induced cell death lacks caspase-3 activation (Supplementary Figure 2). It has been suggested that 3-NP-mediated and excitotoxic cell death represents a caspase-independent, nonapoptotic form of cell death accompanied by calpain and cathepsin activation. ${ }^{35-37}$

Routine mitochondrial fission and fusion cycles ensure mixing of metabolites and mtDNA, determines mitochondrial shape and numbers, and supports bioenergetic functionality. ${ }^{12}$ Mutations in mitochondrial fusion GTPase MFN2 and OPA1, oxidative/nitrosative stress, and neurotoxins, all linked to neurodegeneration, trigger mitochondrial fragmentation. Mitochondrial fusion correlates with increased cell survival, although the mechanism remains elusive. Results of this study suggest that mitochondrial fragmentation, complex II inhibition, NMDA receptor activation, and free radical production might contribute to HD-linked pathogenesis. Mitochondrial fission might amplify neurodegeneration and cell death. That $\mathrm{mtH}$ tt blocks mitochondrial movement and causes formation of punctate mitochondria is of particular significance. ${ }^{38,39}$ Future studies are needed to investigate whether $\mathrm{mtH}$ tt might impair the mitochondrial fission and fusion balance.

\section{Materials and Methods}

Reagents and plasmids. EBSS (Earle's Balanced Salt Solution), Hank's buffer, Glutamax, and penicillin-streptomycin were purchased from Invitrogen. Neurobasal medium, B27, Dulbecco's Modified Eagle Medium (DMEM), and bovine calf serum were purchased from Hyclone. The Amaxa Nucleofector kit (rat neuron 
kit) was obtained from Amaxa, the Cell Titerglo kit from Promega, and AP5 from Tocris. Poly-L-lysine, Hepes, F-12, NAC, anti-MAP2 antibodies (clone HM-2), and 3-NP were obtained from Sigma-Aldrich. All remaining chemicals were obtained from Sigma-Aldrich. Hoechst 33342 and Alexa Fluor 568 goat anti-mouse lgG were purchased from Molecular Probes. zVAD-fmk was obtained from BioMol and the BCA protein assay from PIERCE. The DsRed2-Mito vector was purchased from Clontech and ro-GFP-Mito vector was provided by Dr. Remington (University of Oregon, Eugene, OR, USA).

Cortical neuron preparation and transfection. Primary cortical neuronal cultures were prepared from E17 rat embryos. Cortices were dissected in Hank's buffer, incubated in $0.05 \%$ trypsin for $90 \mathrm{~min}$ at $37^{\circ} \mathrm{C}$, and dissociated mechanically in DMEM, $10 \%$ bovine calf serum, $2 \mathrm{mM}$ glutamine, $25 \mathrm{mM}$ Hepes, $10 \% \mathrm{~F}-12$, and $0.25 \%$ penicillin-streptomycin. Cells in suspension were counted and transfected by electroporation using the Amaxa Nucleofector kit (rat neuron kit) and electroporator device (Amaxa; G-13). Cells were plated on poly-L-lysine $(1 \mathrm{mg} / \mathrm{ml}$ ) coated dishes. After 3-4h, the medium was replaced with Neurobasal medium supplemented with 2\% B27, $2 \mathrm{mM}$ Glutamax, and 1\% penicillin-streptomycin. Cultures were maintained at $37^{\circ} \mathrm{C}$ in a humidified $5 \% \mathrm{CO}_{2}$ atmosphere and fed every 5 days. Experiments were performed after 11-14 days in vitro (DIV) with 3-NP (diluted in EBSS and pH adjusted to 7.4 with $\mathrm{NaOH}$ ).

Image acquisition and processing. Images were acquired using an Axiovert Zeiss $100 \mathrm{M}$ microscope equipped with Plan Apochromat $\times 631.4 \mathrm{NA}$ oil and Plan-neofluor $\times 401.3$ NA oil objectives, a DG-4/Lambda10-2 combo Xe-arc illumination unit (Sutter), Lambda 10-2 emission filter wheel (Sutter), a Sensicam QE cooled CCD camera (PCO AG, Germany), and Metamorph 7.01 software with the Multi-Dimensional Acquisition module (Molecular Devices). Neurons were transfected with the ro-GFP-Mito expression vector. ${ }^{19}$ To assay different treatments simultaneously, cultures were grown on four- or eight-well chambered slides (Lab-Tek II). Growth media was replaced with EBSS supplemented with $10 \mathrm{mM}$ Hepes and mounted onto a heated, motorized stage. 3D images were acquired simultaneously by revisiting 10-20 neurons in each well as previously described..$^{13,40} Z$-series (12 planes at $1 \mu \mathrm{m}$ step size) were dehazed and z-projected using a custom version of Metafluor Analyst (Molecular Devices). Ro-GFP-Mito protein was excited at $403 / 12$ and $490 / 20 \mathrm{~nm}$. Emission was detected at $528 / 36 \mathrm{~nm}$. Increases in oxidation were determined by calculating the $403 / 490$ ratio.

Cell fixation and cell-death assay. Neurons were fixed with $3.7 \%$ formaldehyde and $5 \%$ sucrose in PBS for $20 \mathrm{~min}$ at $37^{\circ} \mathrm{C}$ and washed three times with PBS. This method preserves mitochondrial morphology. Chromatin was stained by incubating fixed samples with Hoechst $33342(1 \mu \mathrm{g} / \mathrm{ml})$ at RT for $5 \mathrm{~min}$. Cells with shrunken soma and condensed chromatin by Hoechst 33342 staining were scored as dead neurons. To assess cell death in Supplementary Figure 2, cultures were immunostained with anti-MAP2 antibodies (Sigma; clone HM-2, ascites fluid; $1: 500)$ and chromatin was stained with Hoechst $33342(1 \mu \mathrm{g} / \mathrm{ml})$. Cells with shrunken soma, neurites containing varicosities, and condensed chromatin by Hoechst 33342 staining were scored as dead neurons. Analyses were performed on triplicate coverslips of three independent experiments.

ATP measurement. Neurons were plated in 96-well plates at $25000 \mathrm{cell} / \mathrm{ml}$ in $100 \mu \mathrm{l}$. At 12 DIV and after 3-NP treatments, ATP levels were determined using a luciferase-based Cell Titer Glo assay kit (Promega). Briefly, after rinsing once with PBS, cells were incubated with Cell Titer Glo reagent for $2 \mathrm{~min}$ and readings were performed with a PolarStar microplate reader (BMG). Results were collected from multiple replicate wells (3-6) in each experiment.

Caspase assay. Caspase enzyme activity was measured of triplicates and in four independent experiments using the EnzChek Caspase-3 assay kit 1 (Molecular Probes) and Z-DEVD-AMC as substrate following the instructions of the manufacturer. Extract protein concentrations were determined with the BCA protein assay (PIERCE) and caspase-3 enzyme activity was measured as increase in fluorescence over time at RT and with a PolarStar microplate reader (BMG).

Statistics. Results are expressed as mean \pm S.E.M. Statistical analysis was performed by one-way ANOVA followed by Fisher's test.
Acknowledgements. We thank Dr. Remington (University of Oregon, Eugene, OR, USA) for his ro-GFP-Mito vector. We thank Dr. Gerencser for advice on image acquisition and processing as well as John Johnson and Viviana DeAssis for technical help. This work was supported by NIH grants R01 NS047456, R01 EY016164, R01 NS055193 (to EB-W), the Philippe Foundation, and the Hereditary Disease Foundation (to $\mathrm{GL}$ ). We apologize to the investigators whose work we could not cite owing to space restrictions. We thank Adam Wilson for help with illustrations.

1. Browne SE, Bowling AC, MacGarvey U, Baik MJ, Berger SC, Muqit MM et al. (1997) Oxidative damage and metabolic dysfunction in Huntington's disease: selective vulnerability of the basal ganglia. Ann Neurol 41: 646-653.

2. Gu M, Gash MT, Mann VM, Javoy-Agid F, Cooper JM, Schapira AH (1996) Mitochondrial defect in Huntington's disease caudate nucleus. Ann Neurol 39: 385-389.

3. Panov AV, Gutekunst CA, Leavitt BR, Hayden MR, Burke JR, Strittmatter WJ et al. (2002) Early mitochondrial calcium defects in Huntington's disease are a direct effect of polyglutamines. Nat Neurosci 5: 731-736.

4. Seong IS, Ivanova E, Lee JM, Choo YS, Fossale E, Anderson M et al. (2005) HD CAG repeat implicates a dominant property of huntingtin in mitochondrial energy metabolism. Hum Mol Genet 14: 2871-2880.

5. Tabrizi SJ, Workman J, Hart PE, Mangiarini L, Mahal A, Bates G et al. (2000) Mitochondrial dysfunction and free radical damage in the Huntington R6/2 transgenic mouse. Ann Neurol 47: $80-86$.

6. Bossy-Wetzel E, Petrilli A, Knott AB (2008) Mutant huntingtin and mitochondrial dysfunction. Trends Neurosci 31: 609-616.

7. Benchoua A, Trioulier Y, Zala D, Gaillard MC, Lefort N, Dufour N et al. (2006) Involvement of mitochondrial complex II defects in neuronal death produced by $\mathrm{N}$-terminus fragment of mutated huntingtin. Mol Biol Cell 17: 1652-1663.

8. Jenkins BG, Koroshetz WJ, Beal MF, Rosen BR (1993) Evidence for impairment of energy metabolism in vivo in Huntington's disease using localized 1H NMR spectroscopy. Neurology 43: 2689-2695.

9. Milakovic T, Quintanilla RA, Johnson GV (2006) Mutant huntingtin expression induces mitochondrial calcium handling defects in clonal striatal cells: functional consequences. J Biol Chem 281: 34785-34795.

10. Choo YS, Johnson GV, MacDonald M, Detloff PJ, Lesort M (2004) Mutant huntingtin directly increases susceptibility of mitochondria to the calcium-induced permeability transition and cytochrome c release. Hum Mol Genet 13: 1407-1420.

11. Brouillet E, Conde F, Beal MF, Hantraye P (1999) Replicating Huntington's disease phenotype in experimental animals. Prog Neurobiol 59: 427-468.

12. Knott AB, Perkins G, Schwarzenbacher R, Bossy-Wetzel E (2008) Mitochondrial fragmentation in neurodegeneration. Nat Rev Neurosci 9: 505-518.

13. Barsoum MJ, Yuan H, Gerencser AA, Liot G, Kushnareva Y, Graber S et al. (2006) Nitric oxide-induced mitochondrial fission is regulated by dynamin-related GTPases in neurons. EMBO J 25: 3900-3911.

14. Yuan H, Gerencser AA, Liot G, Lipton SA, Ellisman M, Perkins GA et al. (2007) Mitochondrial fission is an upstream and required event for bax foci formation in response to nitric oxide in cortical neurons. Cell Death Differ 14: 462-471.

15. Milakovic T, Johnson GV (2005) Mitochondrial respiration and ATP production are significantly impaired in striatal cells expressing mutant huntingtin. $J$ Biol Chem 280 30773-30782.

16. Chang DT, Reynolds IJ (2006) Differences in mitochondrial movement and morphology in young and mature primary cortical neurons in culture. Neuroscience 141: 727-736.

17. Jahani-AsI A, Cheung EC, Neuspiel M, MacLaurin JG, Fortin A, Park DS et al. (2007) Mitofusin 2 protects cerebellar granule neurons against injury-induced cell death. J Biol Chem 282: 23788-23798.

18. Rintoul GL, Filiano AJ, Brocard JB, Kress GJ, Reynolds IJ (2003) Glutamate decreases mitochondrial size and movement in primary forebrain neurons. J Neurosci 23: 7881-7888.

19. Hanson GT, Aggeler R, Oglesbee D, Cannon M, Capaldi RA, Tsien RY et al. (2004) Investigating mitochondrial redox potential with redox-sensitive green fluorescent protein indicators. J Biol Chem 279: 13044-13053.

20. Brouillet E, Jacquard C, Bizat N, Blum D (2005) 3-Nitropropionic acid: a mitochondrial toxin to uncover physiopathological mechanisms underlying striatal degeneration in Huntington's disease. J Neurochem 95: 1521-1540.

21. Karanian DA, Baude AS, Brown QB, Parsons CG, Bahr BA (2006) 3-Nitropropionic acid toxicity in hippocampus: protection through $\mathrm{N}$-methyl-D-aspartate receptor antagonism. Hippocampus 16: 834-842.

22. Nasr P, Gursahani HI, Pang Z, Bondada V, Lee J, Hadley RW et al. (2003) Influence of cytosolic and mitochondrial $\mathrm{Ca} 2+$, ATP, mitochondrial membrane potential, and calpain activity on the mechanism of neuron death induced by 3-nitropropionic acid. Neurochem Int 43: 89-99.

23. Fink SL, Ho DY, Sapolsky RM (1996) Energy and glutamate dependency of 3-nitropropionic acid neurotoxicity in culture. Exp Neurol 138: 298-304

24. Pang Z, Geddes JW (1997) Mechanisms of cell death induced by the mitochondrial toxin 3-nitropropionic acid: acute excitotoxic necrosis and delayed apoptosis. J Neurosci 17: 3064-3073. 
25. Lee WT, Yin HS, Shen YZ (2002) The mechanisms of neuronal death produced by mitochondrial toxin 3-nitropropionic acid: the roles of $\mathrm{N}$-methyl-D-aspartate glutamate receptors and mitochondrial calcium overload. Neuroscience 112: 707-716.

26. Zeron MM, Fernandes HB, Krebs C, Shehadeh J, Wellington CL, Leavitt BR et al. (2004) Potentiation of NMDA receptor-mediated excitotoxicity linked with intrinsic apoptotic pathway in YAC transgenic mouse model of Huntington's disease. Mol Cell Neurosci 25: 469-479.

27. Wang J, Green PS, Simpkins JW (2001) Estradiol protects against ATP depletion mitochondrial membrane potential decline and the generation of reactive oxygen species induced by 3-nitroproprionic acid in SK-N-SH human neuroblastoma cells. J Neurochem 77: 804-811.

28. Mandavilli BS, Boldogh I, Van Houten B (2005) 3-nitropropionic acid-induced hydrogen peroxide, mitochondrial DNA damage, and cell death are attenuated by Bcl-2 overexpression in PC12 cells. Brain Res Mol Brain Res 133: 215-223.

29. Indo HP, Davidson M, Yen HC, Suenaga S, Tomita K, Nishii T et al. (2007) Evidence of ROS generation by mitochondria in cells with impaired electron transport chain and mitochondrial DNA damage. Mitochondrion 7: 106-118.

30. Cepeda C, Hurst RS, Calvert CR, Hernandez-Echeagaray E, Nguyen OK, Jocoy E et al. (2003) Transient and progressive electrophysiological alterations in the corticostriatal pathway in a mouse model of Huntington's disease. J Neurosci 23: 961-969.

31. Behrens PF, Franz P, Woodman B, Lindenberg KS, Landwehrmeyer GB (2002) Impaired glutamate transport and glutamate-glutamine cycling: downstream effects of the Huntington mutation. Brain 125: 1908-1922.
32. Shin JY, Fang ZH, Yu ZX, Wang CE, Li SH, Li XJ (2005) Expression of mutant huntingtin in glial cells contributes to neuronal excitotoxicity. J Cell Biol 171: 1001-1012.

33. Cui L, Jeong H, Borovecki F, Parkhurst CN, Tanese N, Krainc D (2006) Transcriptional repression of PGC-1alpha by mutant huntingtin leads to mitochondrial dysfunction and neurodegeneration. Cell 127: 59-69.

34. Wang G, Moniri NH, Ozawa K, Stamler JS, Daaka Y (2006) Nitric oxide regulates endocytosis by S-nitrosylation of dynamin. Proc Natl Acad Sci USA 103: 1295-1300.

35. Bizat N, Galas MC, Jacquard C, Boyer F, Hermel JM, Schiffmann SN et al. (2005) Neuroprotective effect of ZVAD against the neurotoxin 3-nitropropionic acid involves inhibition of calpain. Neuropharmacology 49: 695-702.

36. Galas MC, Bizat N, Cuvelier L, Bantubungi K, Brouillet E, Schiffmann SN et al. (2004) Death of cortical and striatal neurons induced by mitochondrial defect involves differential molecular mechanisms. Neurobiol Dis 15: 152-159.

37. Kroemer G, Galluzzi L, Vandenabeele P, Abrams J, Alnemri ES, Baehrecke EH et al. (2009) Classification of cell death: recommendations of the Nomenclature Committee on Cell Death 2009. Cell Death Differ 16: 3-11.

38. Chang DT, Rintoul GL, Pandipati S, Reynolds IJ (2006) Mutant huntingtin aggregates impair mitochondrial movement and trafficking in cortical neurons. Neurobiol Dis 22: 388-400.

39. Trushina E, Dyer RB, Badger II JD, Ure D, Eide L, Tran DD et al. (2004) Mutant huntingtin impairs axonal trafficking in mammalian neurons in vivo and in vitro. Mol Cell Biol 24: 8195-8209.

40. Song W, Bossy B, Martin OJ, Hicks A, Lubitz S, Knott AB et al. (2008) Assessing mitochondrial morphology and dynamics using fluorescence wide-field microscopy and 3D image processing. Methods 46: 295-303.

Supplementary Information accompanies the paper on Cell Death and Differentiation website (http://www.nature.com/cdd) 